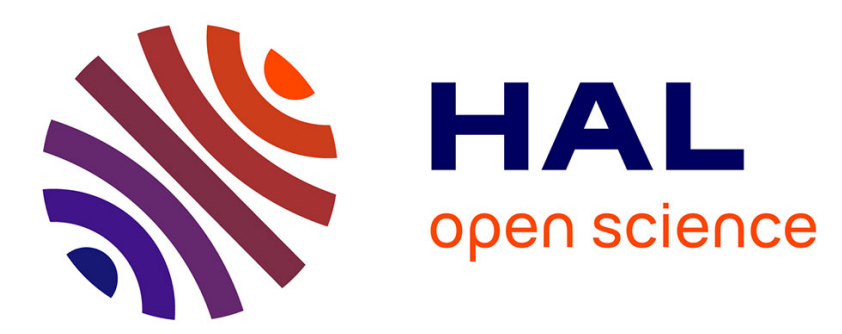

\title{
The mirror neuron system: How cognitive functions emerge from motor organization
}

Leonardo Fogassi

\section{To cite this version:}

Leonardo Fogassi. The mirror neuron system: How cognitive functions emerge from motor organization. Journal of Economic Behavior and Organization, 2010, 77 (1), pp.66. 10.1016/j.jebo.2010.04.009 . hal-00921186

\section{HAL Id: hal-00921186 \\ https://hal.science/hal-00921186}

Submitted on 20 Dec 2013

HAL is a multi-disciplinary open access archive for the deposit and dissemination of scientific research documents, whether they are published or not. The documents may come from teaching and research institutions in France or abroad, or from public or private research centers.
L'archive ouverte pluridisciplinaire HAL, est destinée au dépôt et à la diffusion de documents scientifiques de niveau recherche, publiés ou non, émanant des établissements d'enseignement et de recherche français ou étrangers, des laboratoires publics ou privés. 


\section{Accepted Manuscript}

Title: The mirror neuron system: How cognitive functions emerge from motor organization

Author: Leonardo Fogassi

PII: $\quad$ S0167-2681(10)00177-0

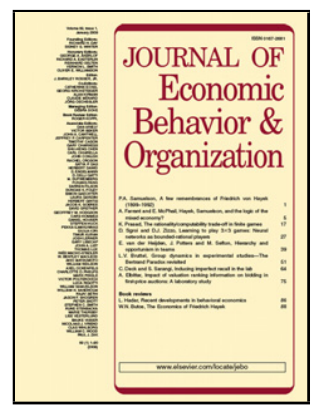

DOI: doi:10.1016/j.jebo.2010.04.009

Reference: $\quad$ JEBO 2604

To appear in: Journal of Economic Behavior \& Organization

Received date: $\quad$ 26-7-2009

Revised date: $\quad 19-4-2010$

Accepted date: $\quad$ 20-4-2010

Please cite this article as: Fogassi, L., The mirror neuron system: How cognitive functions emerge from motor organization, Journal of Economic Behavior and Organization (2010), doi:10.1016/j.jebo.2010.04.009

This is a PDF file of an unedited manuscript that has been accepted for publication. As a service to our customers we are providing this early version of the manuscript. The manuscript will undergo copyediting, typesetting, and review of the resulting proof before it is published in its final form. Please note that during the production process errors may be discovered which could affect the content, and all legal disclaimers that apply to the journal pertain. 
The mirror neuron system:

How cognitive functions emerge from motor organization

\author{
Leonardo Fogassi*
}

\begin{abstract}
In the cortical motor system, matching between motor representations and sensory inputs allows the emergence of different types of cognitive abilities. One of these matching mechanisms is represented by monkey mirror neurons that activate both when a monkey executes a goal-related motor act and when it observes a similar motor act performed by another individual. The mirror neuron matching system that probably underlies action understanding has been demonstrated also in humans. In this article the main features of mirror neurons and the findings demonstrating their relevance for intention and emotion understanding in humans will be described and discussed.
\end{abstract}

"Fogassi's address: Department of Neurosciences, Department of Psychology, Italian Institute of Technology, University of Parma, Italy. Email: leonardo.fogassi@ unipr.it. Homepage: http://www.unipr.it/arpa/mirror/english/staff/fogassi.htm. 


\subsection{Introduction}

The notion that we possess a kind of immediate understanding of what others are doing or feeling is quite old. Adam Smith, for example, proposed the concept of sympathy, according to which we are capable of feeling something similar to what others feel by simply observing others' behaviour (Smith, 1976). Many of his descriptions of the attitude of a person observing the actions and emotions of others resemble the notion of empathy. Although nowadays this notion is often used to indicate the capacity to share others' feelings, Lipps (1903) introduced this term (einfuhlung) in aesthetics to indicate the attitude of an observer in front of an artist's work. Note that in his original definition the observer is not looking at another individual. However, one can easily imagine that this attitude becomes even more pronounced when we are in front of other persons acting or feeling emotions. Although it is known that, at least for emotions, people "empathize" with different degrees of involvement, nobody can deny that we share this capacity with all healthy individuals of our species.

A very important issue about empathy concerns the underlying neurophysiological mechanisms. One hypothesis is that higher order sensory elaboration, together with memory retrieval of known biological stimuli, can account for this type of knowledge. A further elaboration of this theory is that empathy relies on some type of inferential process, like reasoning. This type of view would assign a major role to associative cortical regions that would receive high order sensory information and transform it into perception. A possible role of internal motor representations in this process would be virtually excluded. An opposite theory postulates that understanding actions and the internal states of others can be achieved through an automatic process that matches the biological sensory input with the internal motor knowledge of the observer. According to this view internal motor representations play a crucial role, because they represent the personal knowledge through which every individual assigns meaning to the external world, in this case to the biological stimuli present in it. 
The two theories about the possible neural mechanisms underlying empathy can be included in a more general theoretical framework concerning the way in which information is processed by the cerebral cortex. A traditional view, that will be referred to as "serial", maintained that at the cortical level incoming sensory information is processed through a series of hierarchical steps involving areas of increasing complexity, belonging to the posterior half of the cortex. The final outcome of this processing consists in the achievement of different types of percepts (e.g. object or space perception). According to this serial view, the motor cortex (located in the frontal lobe) would receive this high-level outcome in order to execute various types of motor behavior related to these sensory percepts. For example, once visual space has been coded, this information is used by the motor system to command arm or body movements directed to different space locations. In this view, the motor cortex would represent the last stage of information processing, playing mostly an executive role.

The neuroanatomical data of the last two decades clearly challenges the serial view and has prompted a different perspective that we will refer to as "parallel" and that better explains the way in which information is processed by the cerebral cortex. Most of the connections between posterior and anterior cortical areas are reciprocal, thus indicating that the flow of information runs in parallel, leading to a strict reciprocal influence between action and perception (see also MolnarSakacs, 2010). Furthermore, single neuron recording data demonstrate that the motor cortex, far from being a purely executive cortical sector, contains stored representations of the goal of motor acts. These representations are provided (through the above mentioned neuroanatomical connections) to the posterior cortical areas, and the role of these motor signals is that of giving meaning to incoming sensory information. Thus, our understanding of the external world is, at least partly, based on the automatic activation of the motor system. There are several examples of this type of influence of the motor system on perception. One of these is by Craighero et al. (1999), who demonstrated that if a subject is instructed to grasp a bar using a specific type of wrist orientation, the reaction time to the subsequent presentation, on a monitor, of a bar with a congruent orientation 
will be faster than when a bar of different orientation is presented. The same effect occurs when the experiment is run in the opposite order (first observation of a stimulus, then grasp in a congruent or incongruent way) (Craighero et al. 1996). This suggests that action and perception are, strictly, influencing one another.

The aim of this paper is to review the evidence of mirror neurons (MNs) in the monkey and show how these findings challenge the traditional view of how the brain processes information stimuli and organizes motor action. It should become clear that empathy, which has been assumed to be a replication of the reasoning of the observed individual, actually involves motor knowledge. Section 1.2 discusses in detail the traditional view and how it is challenged by the new MNs discoveries. Section 2.1 explicates the specialization of MNs into "strictly congruent" and "broadly congruent" neurons. Section 2.2 reviews the interesting findings on how mirror neurons also specialize with respect to spatial and social distance. Section 2.3 discusses the mirror neuron system (MNS) in humans, which is of interest with regard to emotional empathy. Section 3.1 deals with the findings that MNS is sensitive to the function of "understanding," called also "mind reading" and "cognitive empathy." Section 3.2 focuses on the understanding of the emotion of the observed person. Section 4 deals with Adam Smith's thesis that sympathy involves not only the simulation or mimicking of the observed affective state, but also involves approval or critical evaluation. Section 5 concludes.

\subsection{Parieto-premotor cortical circuits connect motor representations and sensory}

\section{inputs}

As stated in the Introduction, according to the serial view, cortical processing of information is directed from posterior cortical sectors to the frontal ones (see Goodale and Milner 1992). In the last twenty years, neuroanatomical studies demonstrated a different process, that might be called parallel: there are several, dedicated parieto-frontal circuits, each serving a specific type of sensorimotor transformation (Pandya and Seltzer 1982; Rizzolatti et al. 1998; Rizzolatti and 
Luppino 2001; Rozzi et al. 2006). For example, there is a circuit for grasping, one for reaching, another for goal-directed eye movements. The frontal and the parietal nodes of each of these circuits are reciprocally connected. This finding of course indicates that there is no rigid separation between perceptual and motor properties. It rather points to a sharing of these functions inside each parietofrontal circuit. Moreover, as it will become clearer below, these circuits are not only used for sensorimotor transformations, but provide the basis for the emergence of different types of cognitive function.

According to the serial view, the main aim of the motor cortex would be that of coding movement parameters, such as force, amplitude and direction. However, many neurophysiological studies have shown that the main function of the motor cortex, in particular of premotor areas, is that of coding goal directed motor acts (that is movements directed to a target, see Rizzolatti et al. 2004), while primary motor cortex and subcortical centres would translate these goals in actual execution. In fact, single neuron studies carried out in the ventral premotor cortex of the macaque monkey (areas F4 and F5, illustrated in Figure 1, see Matelli et al. 1985) showed that these areas code several types of motor act executed with axial (trunk), proximal (arm) and distal (hand and mouth) effectors (Gentilucci et al. 1988; Rizzolatti et al. 1988; Fogassi et al. 1996; Ferrari et al. 2003).

- Figure 1 about here -

In particular, single neurons of area F5 activate when a monkey executes goal-related hand and mouth motor acts (Rizzolatti et al. 1988) such as grasping, manipulating, holding, tearing objects. Grasping neurons constitute the most represented category. The level of abstraction reached by these neurons is demonstrated by the finding that there are grasping neurons responding when the monkey grasps food with the left hand, with the right hand or with the mouth. Neurons of this kind cannot be related to the execution of simple movements. They must code the motor act "grasp" in an abstract way. Actually, they seem to reflect the property - well known at the level of whole 
individual - that when we think to grasp something we can do it with the hand, with the mouth or even with the foot. The concept of goal coding applied to these neurons have been recently further corroborated by a study (Umiltà et al. 2008), in which F5 grasping neurons were recorded in monkeys trained to grasp objects using two different tools: "normal pliers" and "reverse pliers". Interestingly, these tools require opposite movements to grasp an object. The results showed that the same neurons become activated not only during grasping a piece of food with the hand, but also when food was grasped with the tools, regardless of whether during this act the hand closed (normal pliers) or opened (reverse pliers), thus confirming that these neurons code the goal of the motor act.

Area F5 neurons code not only the abstract goal of a motor act, but also more specific goals. For example, there are neurons that activate when the monkey grasps a small object using a precision grip, independently of the hand used to perform it, but not when it grasps a larger object using a power grasp.

Altogether, these findings allow us to conclude that in area F5 there is a motor "vocabulary" (see Rizzolatti et al. 2004) in which the "words" are represented by categories of neurons coding motor acts at different levels of abstraction. This vocabulary, partly innate and partly refined during development, represents the individual's "internal motor knowledge".

The goal-related features of F5 neurons are not limited to this area, because similar properties are shown also by neurons of an area located in the parietal cortex, namely area PFG (see Figure 1), which is reciprocally connected with area F5 (Rozzi et al. 2006, Rozzi et al. 2008). This enables us to conclude that there is a motor circuit for the representation of hand and mouth goalrelated motor acts. Such an organization makes the motor vocabulary addressable by several sensory inputs. Thanks to this connection, these inputs receive their meaning from the motor knowledge stored in the motor vocabulary. One of these inputs is that concerning biological actions, such as hand and mouth actions performed by other individuals. This type of sensory-to-motor connection allows the phylogenetic and ontogenetic formation of MNs. 


\subsection{Mirror neurons in monkeys}

MNs are a category of F5 visuomotor neuron. Their name depends on the fact that they respond in two situations: when a monkey performs a hand motor act and when it observes another individual (a human being or another monkey) performing the same or a similar motor act. Typically, they do not respond to simple object presentation or when the observed motor act is mimicked in absence of the target (Gallese et al. 1996; Rizzolatti et al. 1996). On the visual response side, the observed motor acts that are more frequently effective in triggering mirror neuron visual responses are grasping, manipulating, tearing and holding objects. Some of these neurons are very specialized, since, beyond coding the type of goal-directed motor act, they code also the type of observed grip. Besides hand-related MNs there are also mouth-related MNs, which respond to the observation of goal-related motor acts performed with the mouth. The most represented effective mouth motor act is grasping (Ferrari et al. 2003).

Since the visual response of MNs is related to hand-object interaction, one can reach the general conclusion that they code goal-directed motor acts performed by others. This type of coding does not necessarily require the full vision of the other's action. When we see somebody else doing something and part of his action is hidden behind an occluder, we are nonetheless able to recognize what the other is doing. A study carried out some years ago addressed the issue of whether MNs become active also during the observation of partially hidden motor acts (Umiltà et al. 2001). In this study, in one condition the monkey observed a fully visible motor act directed toward a visible object ("full vision" condition), while in the other it observed the same motor act, but its final part (hand-object interaction) was hidden behind an occluding screen ("hidden" condition). In this latter condition the monkey first saw the object and then this was hidden behind the screen; then, with very short delay, the hand of the experimenter, starting its movement in full vision, disappeared behind the screen and executed the same motor act as in the "full vision" condition. The majority of F5 MNs tested with this paradigm responded to the observation of hand motor acts even when the final part of these acts, that triggered the response in full vision, was hidden from the monkey. Very 
likely this neuronal response is built by keeping in memory the previously presented object and by reconstructing the missing part of the observed motor act. This reconstruction is possible only if the monkey's motor cortex contains a representation of the whole act, including the knowledge of its consequences. According to the serial view, this would have been unlikely, because in this case action perception would rely on its full visual description.

When we cannot see an action but can only hear its sound (in the case of noisy actions) we are generally able to understand the action goal. This capacity is directly exemplified by audiovisual MNs, a subcategory of F5 MNs. They activate when a monkey not only observes, but also hears the sound of a motor act (Kohler et al. 2002). The response of these neurons is specific for the type of motor act seen and heard. For example, monkeys respond to peanuts breaking when the act is only observed, only heard, or both heard and observed, and do not respond to the vision and sound of another act, or to non-specific sounds. The presence of audio-visual MNs demonstrates that the motor vocabulary can be addressed also from an acoustic input related to biological actions and give these neurons the capacity to code the goal of that particular motor act. These features are very well accommodated by a parallel view, in which motor representations are used to provide meaning, in this case to the acoustic input. In addition, note that the property of understanding a content independently of its modality, be it visual or acoustic, is typical of language.

All MNs, by definition, have motor properties and these properties normally match very well their visual properties. However, an analysis of the congruence between the visual and the motor responses effective in activating MNs showed that they fall into two major categories: "strictly congruent," i.e., specialized, and "broadly congruent," i.e., general, neurons. Neurons belonging to the first category show a perfect coincidence between the observed and executed motor act effective in eliciting the neuron response. For example, a neuron can activate only during execution and observation of a precision grip, but not during execution and observation of a power grasp. Neurons belonging to the second category show congruence in terms of the general goal that is coded, but often can show some difference between the executed and observed effective motor 
act. For example, a neuron can activate only during execution of a precision grip, but on the visual side can activate during observation of both precision grip and power grasp. Thus, this latter neuron codes a grasping motor act, but the motor response is more specialized than the visual one. The percentage of occurrence of neurons of the two categories is about $30 \%$ and $60 \%$, respectively. The remaining $10 \%$ do not show a direct relation between visual and motor response. These percentages can probably be interpreted in the light of behavioral findings related to imitative processes. It is well established that monkeys do not show true imitation, that is, the immediate reproduction of a novel observed act performed by another individual. However, they show a certain number of imitative phenomena, like neonatal imitation (limited to a few days from birth, see Ferrari et al. 2006) and eating facilitation (the increase in eating behavior of individuals observing other conspecifics that are involved in this type of behavior, see Ferrari et al. 2005). These processes involve innate or early-achieved behaviors. Because of their strict similarity with the observed behavior they reproduce, it is very likely that they are underpinned by a mirror neuron mechanism. When the behavior reproduces exactly the model, as in neonatal imitation, strictly congruent MNs could be involved, while when the model elicits a more complex behavior, although the final goal is the same, as in the case of eating facilitation, broadly congruent or even non congruent MNs could be involved. Independently of whether these degrees of congruence can explain or not these behavioral phenomena, the most likely function of MNs in monkeys is to understand motor acts. This capacity would be automatically triggered by the sight of another individual's motor act and would be grounded on the presence of a motor vocabulary. In other words, our motor knowledge is used to automatically understand what others are doing, as it would be predicted by the parallel view. Note that this notion does not necessarily entail conscious reasoning (see below the discussion on the mirror neuron system in humans).

More recently (Gallese et al. 2002, Rozzi et al. 2008) MNs have been described also in the inferior parietal cortex, mostly in area PFG (Gregoriou et al. 2006), although some are present also in area PF (see Figure 1). The features of these neurons are indistinguishable from those of F5 MNs, 
except for some differences in the categories of motor act that trigger their visual responses. For example, in PFG there are more MNs activated by the observation of the interaction between two hands than in F5. This is interesting, because it suggests an extension of these responses to triadic interactions. Also in terms of the congruence between visual and motor response the percentages of strictly and broadly congruent MNs are almost identical to those described for F5 MNs. In conclusion, F5 and PFG constitute the two main nodes, in the monkey, of the mirror neuron system (MNS). The visual input to this system comes very likely from a region inside the superior temporal sulcus, which is reciprocally connected with PFG, but not with F5. Neurons of this region only respond to observation of hand motor acts, but do not show any motor response (Perrett et al. 1989). Therefore, although necessary for providing the sensory input for the formation of the mirror neuron system, they lack the main property that provides meaning to this input.

\subsection{Coding of the social distance by mirror neurons}

The basic studies on MNs showed that the majority of them are invariant with respect to many factors such as the hand used by the observed agent, the action direction and the space in which the observed action occurred. More recently, however, an interesting space-dependent modulation of the MNs visual response has been shown (Caggiano et al. 2009). In this study, monkeys were trained to perform a motor task in which they were required to grasp objects of different size. Then, a visual condition was run, in which the experimenter performed a grasping act at various distances from the monkey. In some cases the motor act was performed within the monkey's reaching space (peripersonal space), in other cases outside it (extrapersonal space). The results showed that fifty percent of the MNs recorded with this paradigm were sensitive to the distance at which the agent performed the observed motor act. Half of the MNs responded more when the observed motor act was performed close to the monkey (peripersonal neurons), the other half when the observed motor was performed far from the monkey (extrapersonal neurons). Even more interestingly, when a barrier was put in front of the monkey, thus impairing any potential interaction of the monkey 
inside its working space, neurons that responded only in the extrapersonal space begun to activate also in the space close to the monkey, while those responding only in the peripersonal space stopped their response. Half of the MNs tested with this variation of the paradigm showed this property, while the other half were not influenced by it. The result of this second experiment is not contradictory with the first, because in the half of MNs showing a distance-dependent modulation, this is further influenced by the possibility or not for the monkey to interact in its peripersonal, working space. Therefore, when a barrier is introduced, the former peripersonal space becomes, for the monkey, extrapersonal. In the other half, modulation is related only to a metric, fixed coding of the space in which the motor act is performed. These results confirm that space is coded in a motor format (see Fogassi et al. 1996, Rizzolatti et al. 1997), and that this motor code directly influences the perception of other actions, in agreement with the parallel view of cortical information processing.

This modulation of space-dependent MNs can be explained in terms of the interactions that monkeys can have in space. Hence, MNs selective for motor acts performed close to the monkey can be used for competitive behaviour or, if one extrapolates this result to humans, also for cooperative behaviour. MNs selective for motor acts performed far from the monkey can signal the necessity of an approaching behaviour to subsequently interact with the agent performing the observed act. These newly discovered properties appear to be important because they suggest that mirror responses are not exclusively related to action understanding but can trigger subsequent behaviour in response to the observed one.

\subsection{The mirror neuron system in humans}

Nobody doubts that we, as humans, are very good at recognizing and understanding other people's actions. Additionally, we well know our capacity to understand or at least to guess, on the basis of the observed behaviour, what are the intentions and emotions of others. Furthermore, we use this capacity also for orienting our actions in response to the observed behaviour of others. Typical 
examples are imitation, in which we try to reproduce an observed behaviour, normally for learning purposes, or the behaviour involved in many sports or martial arts. It is very likely that most of these functions have, at their basis, a mirror neuron mechanism. Thus, the neuroscientific investigation of the MNS in humans is crucial to understanding the bases of these functions. While Molnar- Szakacs (2010) provides a description of the studies on MNS in humans, a brief summary is in order.

- The presence of a MNS in humans has been demonstrated with electrophysiological and brain imaging techniques (for a review see Rizzolatti and Craighero 2004). Of course, ethical reasons do not allow us to provide a direct demonstration at the level of single neurons. It is possible that in the future we will be able to collect this type of evidence, probably in neurosurgical or epileptic patients, by use of more sophisticated techniques. It must be noted that some researchers, by using as an argument the absence of a direct evidence of the presence of MNs in humans at the single neuron level, hypothesize that although the very same regions activate both during action observation and action execution, it could still be possible that these two responses are due to two different populations, sensory and motor, respectively (see Dinstein 2008). However, it is very unlikely that when evolution has achieved a very adaptive mechanism, such as the observation/execution matching system, it would then be lost at later stages of evolution in the same lineage (primates).

- The presence of the MNS is also corroborated by the fact that its main nodes are in sectors (anteriorly in inferior frontal gyrus and premotor cortex, posteriorly in the inferior parietal lobule) that are considered homologous to the areas forming the mirror neuron system in monkeys (see for example Grafton et al. 1996; Iacoboni et al. 1999; Buccino et al. 2001; Iacoboni et al. 2005).

- One of the sectors belonging to the frontal node of the MNS co-extends with Broca's area, classically known for its involvement in language production. This finding suggests a possible relation between the MNS and language comprehension (Rizzolatti and Arbib 1998; Fogassi and Ferrari 2007). 
- The MNS in humans is involved in functions that are probably absent or barely present in monkeys, such as imitation, recognition of intransitive (devoid of a target object) and symbolic gestures (see also Khalil 2010), and some aspects of language recognition.

Furthermore, there exists a kind of MNS in humans for emotions understanding, which has not yet been found in monkeys.

Some properties of monkey MNs might have constituted the phylogenetic basis for the emergence, in humans, of more sophisticated functions, such as intention understanding and language evolution. The next section deals with the first function..For the second function, see Rizzolatti and Arbib (1998) and Fogassi and Ferrari (2007)

\subsection{Intention understanding}

Humans are very skilled in recognizing the intentions underlying others' actions. This capacity is one of the components of mindreading. There are different theories that try to explain how we can understand others' intentions (see Gallese and Goldman 1998). According to theory-theory ${ }^{1}$ this occurs through an inferential process, while, according to the simulation theory, observation of another individual acting triggers an internal simulation of her/his actions. While the first theory requires reasoning, the second implies an automatic retrieval of motor representations. The evidence that the motor system, through parallel sensorimotor processing, supports the emergence of cognitive functions, suggests that the understanding of simple actions at least can rely on the automatic retrieval of the corresponding motor representations, as postulated by simulation theory.

\footnotetext{
${ }^{1}$ Theory-theory maintains that the mindreading capacity of ordinary people implies their knowledge of causal laws that, similarly to physical laws, allow one to relate external stimuli to internal states, some internal states with other internal states (for example intentions and decisions) and, finally, internal states with behaviours. Thus, attributing intention emerges from a theoretical reasoning that assumes the existence of these laws. Theory-theory was much applied to developmental studies.
} 
The consequent empirical question is therefore: do MNs have a role in encoding the intention of others? Two recent studies (Fogassi et al. 2005; Bonini et al. 2009) addressed the issue of whether the response of motor and MNs of inferior parietal (IPL) and ventral premotor (PMv) cortices during execution and observation of a motor act (grasping), respectively, can be influenced by the final goal of the action in which the act is embedded. The neurons were tested while the monkey performed a task in which it had to reach and grasp a piece of food located in front of it and bring it to its mouth (Condition 1) or to place it into a container (Condition 2). The results showed that the majority of grasping neurons in IPL discharged differently according to the intended goal of the action in which grasping was embedded. Although the same effect was found in PMv neurons, this result was weaker than in IPL. These data suggest that the activity of IPL and, to a lesser extent, of PMV grasping neurons reflects the intention of the performing agent. The visual response of IPL and PMv MNs was studied with the same task. In this case it was the experimenter that grasped a piece of food and brought it to the mouth, or grasped the same piece of food and placed it into a container. Mirror neuron activity was recorded while the monkey observed the two conditions. The results showed that the majority of IPL MNs were differently activated when the observed grasping motor act was followed by bringing to the mouth or by placing. Furthermore, MNs that discharged more strongly during execution of grasping for eating than during grasping for placing also discharged more strongly during the observation of grasping for eating. Conversely, neurons selective for execution of grasping to place also discharged more strongly during the observation of this motor act.

The general interpretation given to these data is that the motor system is organized in intentional chains of neurons, each dedicated to a specific action goal. When the monkey executes a motor act as part of a particular action, the intensity of a neuron's response depends on whether this neuron belongs to the chain coding that action or not. If this is true, when observing an action, IPL MNs coding a specific motor act embedded in it can recruit the neuronal chain coding a particular 
final goal. This would allow the monkey to predict what is the goal of an observed action and, in this way, "read" the intention of the acting individual.

These findings demonstrate, according to the parallel view of information processing, that inferior parietal and ventral premotor cortex constitute an "intentional" circuit, in which the primary role of IPL is probably that of organizing motor acts in intentional chains, while that of PMv is mainly that of providing the motor acts representations for chain building. Note, however, that both areas share the same function of understanding others' intentions.

A recent fMRI study in humans (Iacoboni et al. 2005; see Molnar-Szakacs 2010) indicates that our species also uses the MNS in order to understand the intentions of others. Thus, it appears that the MNS in monkeys provides the first neural substrate for a primitive understanding of other's intentions, which probably paved the way for the evolution of the more sophisticated aspects of mind reading present in humans. Very likely, many of these aspects still rely on automatic activation of the parieto-frontal mirror neuron circuit.

\subsection{Emotion understanding}

Emotion understanding has been at the core of the empathy issue for many years. The central point here is whether there exists a kind of mirror mechanism for emotions similar to that described for actions and whether this is already present in monkeys. First of all we have to consider that, similarly to the different theories about intention understanding (see above), there are different ways of explaining our understanding of others' feelings. One proposal maintains that emotion understanding occurs through an inferential elaboration based on emotion-related sensory information, with a mechanism similar to that proposed by theory-theory (a theoretical reasoning) to explain intention understanding. For example, a certain facial expression means happiness, another sadness, and so on. Another, very different proposal is that we can understand emotions because emotion-related sensory information is directly mapped onto neural structures that, when active, determine a similar emotional reaction in the observer. This second proposal implies a kind of 
simulation of the affective state of the other individual, involving a partial recruitment of visceromotor associated expressions. Note the similarity with action understanding: only in this latter case a "first person knowledge", that is knowledge based on first person "visceromotor" experience, is activated. In fact, the neural structures related to affective states are responsible for visceromotor outputs, that is motor commands directed to visceral organs. In both monkeys and humans, a cortical structure much involved in this kind of output is the insular cortex.

The insular cortex is the target of fibers conveying information about an individual's internal body state (Craig 2002), in addition to olfactory, taste, somatosensory and visual inputs. The insular cortex is buried in the depth of sylvian fissure, covered by the lateral part of frontal and parietal cortices. Owing to its deep location, this cortical sector is less easy to investigate with microelectrodes for single neuron recordings. In addition, it is quite elongated in the rostro-caudal axis, so that it encompasses areas with different anatomical features. On the motor side, according to what has been shown by quite old data in humans (Penfield and Faulk 1955; Showers and Lauer 1961; see also for more recent demonstration Krolak-Salmon et al. 2003), it has been reported that microstimulation of the monkey insula can produce both somatic and visceral responses (Caruana et al. 2008). One example of the latter is the gesture observed when vomiting. We lack, however, the important information on whether there are neurons in this region responding to both the presentation of emotional expressions and to the production of the same type of expression. In contrast with the discovery of MNs related to actions, human data can provide hints on this issue. The advantage in humans is the possibility of using brain imaging techniques that do not require the insertion of electrodes in this deep structure.

Evidence about involvement of the insula in empathy has been reviewed in the article by Molnar-Szacacs (2010). Therefore, this article describes only a study on a basic emotion that is very important for survival, namely disgust. Brain imaging studies showed that an individual feeling disgust activates both amygdala and insula areas (Augustine 1996; Zald et al. 1998; Zald and Pardo 2000; Royet et al. 2001; Small et al. 2003). In addition, it has been demonstrated that the insula is 
also activated by observation of disgusted facial expressions (Phillips et al. 1997, 1998;

Sprengelmeyer et al. 1998; Schienle et al. 2002). Interestingly, a recent fMRI study by Wicker et al. (2003) addressed the issue of whether understanding of other's disgust could rely on the same neural machinery involved when an individual feels disgust. In this study, participants had to smell pleasant or disgusting odorants and observe video-clips showing actors smelling disgusting, pleasant and neutral odorants and expressing the corresponding emotions. In both conditions disgust evoked activation of limbic structures such as the amygdala, cingulate cortex and anterior insula. Most importantly, the same sectors of anterior insula and, to a lesser degree, anterior cingulate were activated by the exposure to disgusting odorants and by the observation of disgust in others. Interestingly, clinical studies show that insular lesions produce deficits in recognizing disgust expressed by others (Calder et al. 2000; Adolphs et al. 2003). Altogether, these findings suggest that humans understand disgust, and most likely other emotions (see Carr et al. 2003, Singer et al. 2004), through a direct mapping mechanism that recruits a first-person experience of the same feelings that are normally associated with precise visceromotor reactions.

\section{From empathy to sympathy}

The recognition of others' emotions based on first person knowledge is probably not enough for a deep sharing of an emotional state with another individual. However, recognition of others' feelings enhances the sharing of an emotional state with another individual. Adam Smith (1976) discusses many examples of how familiarity enhances sympathy in The Theory of Moral Sentiments. Interestingly, recent neuroscientific literature shows that different levels of "empathy" and/or the relationship between the observer and the observed agent can be reflected by different degrees of neural activation or even by activation of selective neural structures. For instance, Singer and coworkers (2004) performed an fMRI study in which participants were couples. Female partners were scanned, while their male partners were just outside the scanner and only their hands were visible to the female. Two different cues informed the female partner whether she was going to 
receive a light painful stimulus ("self" condition) or that her partner was going to receive it ("other" condition). Among the areas activated in the two conditions, anterior insula and anterior cingulate cortex presented overlapping activations. The empathic scales constructed with the subjects' responses to specific questionnaires revealed that there was a significant positive correlation between the degree of empathy and the intensity of activation in these cortical regions. In a second, similar experiment (Singer et al. 2006), both females and males were scanned while they observed another individual who received painful stimuli. The observed individual was one of two actors who, before scanning, had played a game with subjects (one acted as a fair player, the other as unfair). The results of the experiment were similar to the previous one concerning the females, while in males there was activation of the insula only when they observed the fair player feeling pain; however, when they observed the unfair player feeling pain there was an activation of the nucleus accumbens, a reward-related area. This activation correlated with a desire for retaliation, as assessed in a post-scanning interview. These findings suggest that emotion recognition activates the limbic system, but a) the intensity of activation can vary depending on the degree of "embodiment" of the observed emotion; and b) depending on the context, other areas not directly related to the observed emotion can also be involved.

The problem of moral choice is certainly linked to empathy. Adam Smith, starting from the consideration that we, as observers, resonate with others' actions and emotions, proposes that the moral sense rises from the approval or disapproval of the feelings of others, depending on whether we sympathize or not with their feelings. Thus, also from his considerations one can evince that there are very likely different steps in the sympathetic process. The first is that related to automatic emotion and action recognition, the second is deeper and can involve a larger brain network (see Khalil 2010).

Independently of which mechanisms in humans can explain the different steps of the sympathetic process, it could be interesting to speculate on the evolutionary level reached by monkeys in this respect. From the findings reviewed above, it is possible to claim that in monkeys 
there does exist an automatic system for understanding goal-related motor acts, based on the mirror neuron property of matching observed and executed motor acts. This system, in presence of a well defined context or the memory of recent events, also enables the monkey to understand others' intentions. The activation of this system mainly reflects the activation of our internal knowledge and does not require other, slower, cognitive processes. Note, however, that this activation represents, per se, a motor-based cognitive process.

A mechanism similar to action mirroring can be hypothesized in the monkey emotional realm. The difference from the former (related to motor action) is that, in this case, what is matched is not only a gesture (for example facial expressions or postural adjustments), but also internal states. Thus, at least in part, a different brain network is involved. Note, however, that also in this case motor knowledge is required, grounded in well-established viscero-motor reactions. One can easily suppose that also in this mirroring system, the context and the memory of past events is very important for the modulation of the neuronal activity: a monkey looking at the fearful expression of a conspecific must link it to the stimulus triggering this emotion. Another similarity between action and emotion understanding systems is that both can act as a prelude to specific behavioral responses. In the action system these responses can consist in an automatic mirroring (see Khalil 2010), such as in contagious behavior and in neonatal imitation (Ferrari et al. 2006), or in the triggering of complex behaviors, such as eating facilitation (Ferrari et al. 2005) or various types of competitive behaviors. In the emotional system, instead, this can consist in the elicitation of similar emotional responses in group members or in the triggering of related responses (for example a fearful response to aggressive behavior). Unfortunately, in the monkey, although it is known that many of these responses, at the cortical level, are mediated by cingulate and insular cortex, there is still no indication, at the single neuron level, of a mirroring of the observed emotional behaviors.

There is, however, an example of mirroring in the field of oro-facial communication. It is well known that monkeys display various types of oro-facial expressions, very often related to strong emotional states. There are, however, facial gestures, often accompanied also by specific 
body postures, which are defined as affiliative because they allow positive relations between individuals. Lipsmacking and pucker face are typical affiliative gestures. We found that, within the category of F5 mouth MNs, there is a smaller group of neurons responding to the sight of communicative gestures represented by facial expressions and endowed with affiliative meaning (Ferrari et al. 2003). Although in the lab context it is not always possible to elicit from monkeys communicative reactions other than fear or threat, nonetheless we found MNs responding both when the monkey observed an affiliative gesture (lips protruded face) and when it responded with a related gesture (lipsmacking). This indicates that these neurons not only recognized the specific observed gesture, but also had a role in the appropriate reaction to this gesture.

Affiliative gestures can prelude pleasant interactions between two individuals, such as grooming sessions. However, in monkeys, no instance of consolation behavior, as frequently observed in chimps, has been reported.

\section{Conclusion}

In this article it has been reported that different theories have been advanced for explaining our capacity to understand actions and emotions. One theory is more linked to a traditional cognitive view, grounded on higher order sensory elaboration, perception and the inferential processes that can be built on this type of information. This theory was, at least in part, dependent on a "serial" way of information processing at the cerebral cortex level. An alternative theory is instead grounded on individuals' motor knowledge, as their basis for understanding the social world. This second view is very much connected to a "parallel" view of cortical information processing that supports, on the basis of neuroanatomical and electrophysiological data, the claim that action and perception are reciprocally linked and that motor representations give meaning to the biological sensory input. This concept has received an even stronger support from the discovery of the mirror neuron system, which appears to provide the neural underpinning of an automatic understanding of both actions and emotions. The discharge of MNs, besides understanding, can also be used to trigger several types of 
social behavioral reactions. However, we cannot assign to the MNS the full role of understanding social behavior. In fact, in order to reach further evolutionary steps, such as those of apes and humans, in which somatomotor and affiliative behavior become more sophisticated, areas linked to the MNS must have expanded through evolution to code, in the action context, long-range action planning and, in the emotional context, a higher control of reactions in emotional situations. Studies in humans (see Buccino et al. 2004; Singer et al. 2006; see also Molnar-Szakacs 2010) suggest that areas such as prefrontal and orbitofrontal cortex could be crucial for explaining these increased behavioral functions.

\section{Acknowledgements}

I want to thank the organizers of the Symposium on Emotions, Natural Selection and Rationality, Elias L. Khalil and Jonathan B. Wight, the participants of the Symposium and the University of Richmond for its support. I also want to thank anonymous referees and Istvan Molnar-Szakacs for his very helpful comments on an earlier version of the manuscript. I thank also R. Woods for her help in editing the final version of the manuscript. The usual caveat applies.

\section{References}

Adolphs, R., Tranel, D., Damasio, A.R., 2003. Dissociable neural systems for recognizing emotions. Brain and Cognition 52, 61-69.

Augustine, J.R., 1996. Circuitry and functional aspects of the insular lobe in primates including humans. Brain Research Reviews 22, 229-244.

Bonini, L., Rozzi, S., Ugolotti Serventi, F., Simone, L., Ferrari, P.F., Fogassi, L., 2009. Ventral premotor and inferior parietal cortices make distinct contribution to action organization and intention understanding. Cerebral Cortex doi:10.1093/cercor/bhp200.

Buccino, G., Binkofski, F., Fink, G.R., Fadiga, L., Fogassi, L., Gallese, V., Seitz, R.J., Zilles, K., Rizzolatti, G. Freund, H-J., 2001. Action observation activates premotor and parietal areas in a somatotopic manner: an fMRI study. European Journal of Neuroscience 13, 400-404 . 
Buccino, G., Vogt, S., Ritzl, A., Fink, G.R., Zilles, K., Freund, H.J., Rizzolatti, G., 2004. Neural circuits underlying imitation learning of hand actions: an event-related fMRI study. Neuron 42, 323-334.

Caggiano, V., Fogassi, L., Rizzolatti, G., Thier, P. and Casile, A., 2009. Mirror neurons differentially encode the peripersonal and extrapersonal space of monkeys. Science 324: 403-406.

Calder, A.J., Keane, J., Manes, F., Antoun, N., Young, A.W., 2000. Impaired recognition and experience of disgust following brain injury. Nature Neuroscience 3, 1077-1088.

Carr, L., Iacoboni, M., Dubeau, M.C., Mazziotta, J.C., Lenzi, G.L., 2003. Neural mechanisms of empathy in humans: a relay from neural systems for imitation to limbic areas. Proceedings of the National Academy of Sciences U.S.A 100, 5497-5502.

Caruana, F., Jezzini, A., Rochat, M., Sbriscia Fioretti, B., Stoianov, I., Gentilucci, M., Umiltà, M.A., Rizzolatti, G., Gallese,V., 2008. Intracortical microstimulation mapping of the inner perysilvian regions: effects on behavior and ECG outcome. Society Neuroscience Abstracts 883.5.

Craig, A.D. , 2002. How do you feel? Interoception: the sense of the physiological condition of the body. Nature Reviews Neuroscience 3, 655-666.

Craighero, L., Fadiga, L., Umiltà, C.A., Rizzolatti, G., 1996. Evidence for visuomotor priming effect. Neuroreport 8, 347-9.

Craighero, L., Fadiga, L., Rizzolatti, G., Umiltà, C., 1999. Action for perception: a motor-visual attentional effect. Journal of Experimental Psychology and Human Perception Performance 25, 1673-92.

Dinstein, I., 2008. Human cortex: reflections of mirror neurons. Current Biology 18, R956-959. DOI: 10.1016/j.cub.2008.09.007 
Ferrari, P.F., Gallese, V., Rizzolatti G., Fogassi L., 2003. Mirror neurons responding to the observation of ingestive and communicative mouth actions in the monkey ventral premotor cortex. European Journal of Neuroscience 17,1703-14.

Ferrari, P.F., Maiolini, C., Addess, E., Fogassi, L., Visalberghi, E., 2005. The observation and hearing of eating actions activates motor programs related to eating in macaque monkeys. Behavioural Brain Research 161,95-101.

Ferrari, P.F., Visalberghi, E., Paukner, A., Fogassi, L., Ruggiero, A., Suomi, S.J., 2006. Neonatal imitation in rhesus macaques. PLoS Biology 4,1501-1508.

Fogassi, L., Gallese, V., Fadiga, L., Luppino, G., Matelli, M., Rizzolatti, G., 1996. Coding of peripersonal space in inferior premotor cortex (area F4). Journal of Neurophysiology 76, 141-157.

Fogassi, L., Ferrari, P.F., Gesierich, B., Rozzi, S., Chersi, F., Rizzolatti, G., 2005. Parietal Lobe: from Action Organization to Intention Understanding. Science 308, 662-667.

Fogassi, L., Ferrari, P.F., 2007. Mirror neurons and the evolution of embodied language. Current Directions in Psychological Sciences 16, 136-141.

Gallese, V., Goldman, A., 1998. Mirror neurons and the simulation theory of mind-reading. Trends in Cognitive Sciences 2, 493-501.

Gallese, V., Fadiga, L., Fogassi, L., Rizzolatti, G., 1996. Action recognition in the premotor cortex. Brain 119, 593-609.

Gallese, V., Fadiga, L., Fogassi, L., Rizzolatti, G., 2002. Action representation and the inferior parietal lobule. In: Prinz, W., Hommel, B. (Eds). Common mechanisms in perception and action: Attention and Performance, Vol. XIX. Oxford: Oxford University Press, 334-355. 
Gentilucci, M., Fogassi, L., Luppino, G., Matelli, M., Camarda, R., Rizzolatti, G., 1988.

Functional organization of inferior area 6 in the macaque monkey. I. Somatotopy and control of proximal movements. Experimental Brain Research 71, 475-490.

Goodale, M.A., Milner, A.D., 1992. Separate visual pathways for perception and action. Trends in Neurosciences 15, 20-25.

Grafton, S.T., Arbib, M.A., Fadiga, L., Rizzolatti, G., 1996. Localization of grasp representations in humans by PET: 2. Observation compared with imagination. Experimental Brain Research 112, 103-111.

Gregoriou, G.G., Borra, E., Matelli, M., Luppino, G., 2006. Architectonic organization of the inferior parietal convexity of the macaque monkey. Journal of Comparative Neurology 496, 422451.

Iacoboni, M., Woods, R.P., Brass, M., Bekkering, H., Mazziotta, J.C., Rizzolatti, G. 1999. Cortical mechanisms of human imitation. Science 286, 2526-2528.

Iacoboni, M., Molnar-Szakacs, I., Gallese, V., Buccino, G,, Mazziotta, J.C., Rizzolatti, G., 2005. Grasping the intentions of others with one's own mirror neuron system. PloS Biology 3, 529-535.

Khalil, E., 2010. The Mirror neuron Paradox: How Far is Understanding from Mimicking? Journal of Economic Behavior and Organization .

Kohler, E., Keysers, C., Umiltà, M.A., Fogassi, L., Gallese, V., Rizzolatti, G., 2002. Hearing Sounds, understanding Actions: action representation in mirror neurons. Science 297, 846-48.

Krolak-Salmon, P., Henaff, M.A., Isnard, J., Tallon-Baudry, C., Guénot, M., Vighetto, A., Bertrand, O., Mauguière, F., 2003. An attention modulated response to disgust in human ventral anterior insula. Annals of Neurology 5, 446-453.

Lipps, T. 1903. Ästhetik: Psychologie des Schönen und der Kunst: Grundlegung der Ästhetik, Erster Teil. Hamburg: L Voss.. 
Matelli, M., Luppino, G., Rizzolatti, G., 1985. Patterns of cytochrome oxidase activity in the frontal agranular cortex of the macaque monkey. Behavioural Brain Research 18, 125-136.

Matelli, M., Luppino, G., Rizzolatti, G., 1991. Architecture of superior and mesial area 6 and the adjacent cingulate cortex in the macaque monkey. Journal of Comparative Neurology 311, 445462.

Molnar-Szakacs, I., 2010. From actions to empathy and morality - A neural perspective. Journal of Economic Behavior and Organization .

Pandya, D.N., Seltzer, B., 1982. Intrinsic connections and architectonics of posterior parietal cortex in the rhesus monkey. Journal of Comparative Neurology 204,196-210.

Penfield, W. Faulk, M.E., 1955. The insula: further observations on its function. Brain 78, 445470.

Perrett, D.I., Harries, M.H., Bevan, R., Thomas, S., Benson, P.J., Mistlin, A.J., Chitty, A.K., Hietanen, J.K., Ortega, J.E., 1989. Frameworks of analysis for the neural representation of animate objects and actions. Journal of Experimental Biology 146, 87-113.

Phillips, M.L., Young, A.W., Senior, C., Brammer, M., Andrew, C., Calder, A.J., Bullmore, E.T., Perrett, D.I., Rowland, D., Williams, S.C., Gray, J.A., David, A.S., 1997. A specific neural substrate for perceiving facial expressions of disgust. Nature 389, 495-498.

Phillips, M.L., Young, A.W., Scott, S.K., Calder, A.J., Andrew, C., Giampietro, V., Williams, S.C., Bullmore, E.T., Brammer, M., Gray, J,A., 1998. Neural responses to facial and vocal expressions of fear and disgust. Proceedings of the Royal Society of London Biological Sciences $265,1809-17$.

Rizzolatti, G., Camarda, R., Fogassi, L., Gentilucci, M., Luppino, G., Matelli, M., 1988. Functional organization of inferior area 6 in the macaque monkey: II. Area F5 and the control of distal movements. Experimental Brain Research 71, 491-507. 
Rizzolatti, G., Fadiga, L., Gallese, V., Fogassi, L., 1996. Premotor cortex and the recognition of motor actions. Cognitive Brain Research 3,131-141.

Rizzolatti, G., Fadiga, L., Fogassi, L., Gallese, V., 1997. The space around us. Science 277, 190191.

Rizzolatti, G., Arbib, M.A., 1998. Language within our grasp. Trends in Neuroscience 21, 188-94.

Rizzolatti, G., Luppino, G., Matelli, M., 1998. The organization of the cortical motor system: new concepts. Electroencephalography and Clinical Neurophysiology 106, 283-96.

Rizzolatti, G., Luppino, G., 2001. The cortical motor system. Neuron 31, 889-901.

Rizzolatti, G., Craighero, L., 2004. The mirror-neuron system. Annual Reviews of Neuroscience 27, 169-192.

Rizzolatti, G., Fogassi, L., Gallese, V., 2004. Cortical mechanisms subserving object grasping, action understanding, and imitation. In Gazzaniga M.S. (Ed.). The Cognitive Neurosciences III, Cambridge, USA: MIT Press, 427-440.

Rozzi, S., Calzavara, R., Belmalih, A., Borra, E., Gregoriou, G.G., Matelli, M., Luppino, G., 2006. Cortical Connections of the Inferior Parietal Cortical Convexity of the Macaque Monkey. Cerebral Cortex 16, 1389-1417.

Rozzi, S., Ferrari, P.F., Bonini, L., Rizzolatti, G., Fogassi, L., 2008. Functional organization of inferior parietal lobule convexity in the macaque monkey: Electrophysiological characterization of motor, sensory and mirror responses and their correlation with cytoarchitectonic areas. European Journal of Neuroscience 28, 1569-1588.

Royet, J.P., Hudry, J., Zald, D.H., Godinot, D., Grégoire, M.C., Lavenne, F., Costes, N., Holley, A., 2001. Functional neuroanatomy of different olfactory judgments. Neuroimage 13, 506-19. 
Schienle, A., Stark, R., Walter, B., Blecker, C., Ott, U., Kirsch, P.,, Sammer, G., Vaitl, D., 2002. The insula is not specifically involved in disgust processing: an fMRI study. Neuroreport 13, 20232026.

Showers, M.J.C., Lauer E.W., 1961. Somatovisceral motor patterns in the insula. Journal of Comparative Neurology 117, 107-115.

Singer, T., Seymour, B., O'Doherty, J., Kaube, H., Dolan, R.J., Frith, C.D., 2004. Empathy for pain involves the affective but not the sensory components of pain. Science 303, 1157-1162.

Singer, T., Seymour, B., O'Doherty, J.P., Stephan, K.E., Dolan, R.J., Frith, C.D., 2006. Empathic neural responses are modulated by the perceived fairness of others. Nature 439, 466-469.

Small, D.M., Gregory, M.D., Mak, Y.E., Gitelman, D., Mesulam, M.M., Parrish, T., 2003.

Dissociation of neural representation of intensity and affective valuation in human gustation. Neuron 39, 701-711.

Smith, A. 1976. The Theory of Moral Sentiments. Raphael, D.D., Macfie, A.L. (Eds-). Oxford: Oxford University Press.

Sprengelmeyer, R., Rausch, M., Eysel, U.T., Przuntek, H., 1998. Neural structures associated with recognition of facial expressions of basic emotions. Proceedings of the Royal Society of London Biological Sciences 265, 1927-1931.

Umiltà, M.A., Kohler, E., Gallese, V., Fogassi, L., Fadiga, L., Keyzers, C., Rizzolatti, G., 2001. I know what you are doing: a neurophysiological study. Neuron 31, 155-165.

Umiltà, M.A., Escola, L., Intskirveli, I., Grammont, F., Rochat, M., Caruana, F., Jezzini, A., Gallese, V., Rizzolatti, G., 2008. How pliers become fingers in the monkey motor system. Proceedings of the National Academy of Sciences 105, 2209-2213.

Wicker, B., Keysers, C., Plailly, J., Royet, J.P., Gallese, V., Rizzolatti, G., 2003. Both of us disgusted in my insula: The common neural basis of seeing and feeling disgust. Neuron 40, 655664. 
Zald, D.H., Lee, J.T., Fluegel, K.W., Pardo, J.V., 1998. Aversive gustatory stimulation activates limbic circuits in humans. Brain 121, 1143-1154.

Zald, D.H., Pardo, J.V., 2000. Functional neuroimaging of the olfactory system in humans. International Journal of Psychophysiology 36, 165-181. 


\section{Figure legend}

Figure 1. Subdivisions of motor and posterior parietal cortex of the macaque monkey. Motor areas are classified according to the parcellation of Matelli et al. $(1985,1991)$; the parietal areas are classified according to Pandya and Seltzer (1982) and Gregoriou et al. (2006). F1 corresponds to primary motor cortex, F3 and F6 form the mesial premotor cortex, F2 and F7 the dorsal premotor cortex, F4 and F5 the ventral premotor cortex. PE and PEc form the superior parietal lobule, PF, PFG, PG and Opt the inferior parietal lobule. Abbreviations: AI, inferior arcuate sulcus; AS, superior arcuate sulcus; C, central sulcus; IP, intraparietal sulcus; L, lateral fissure; P, principal sulcus.

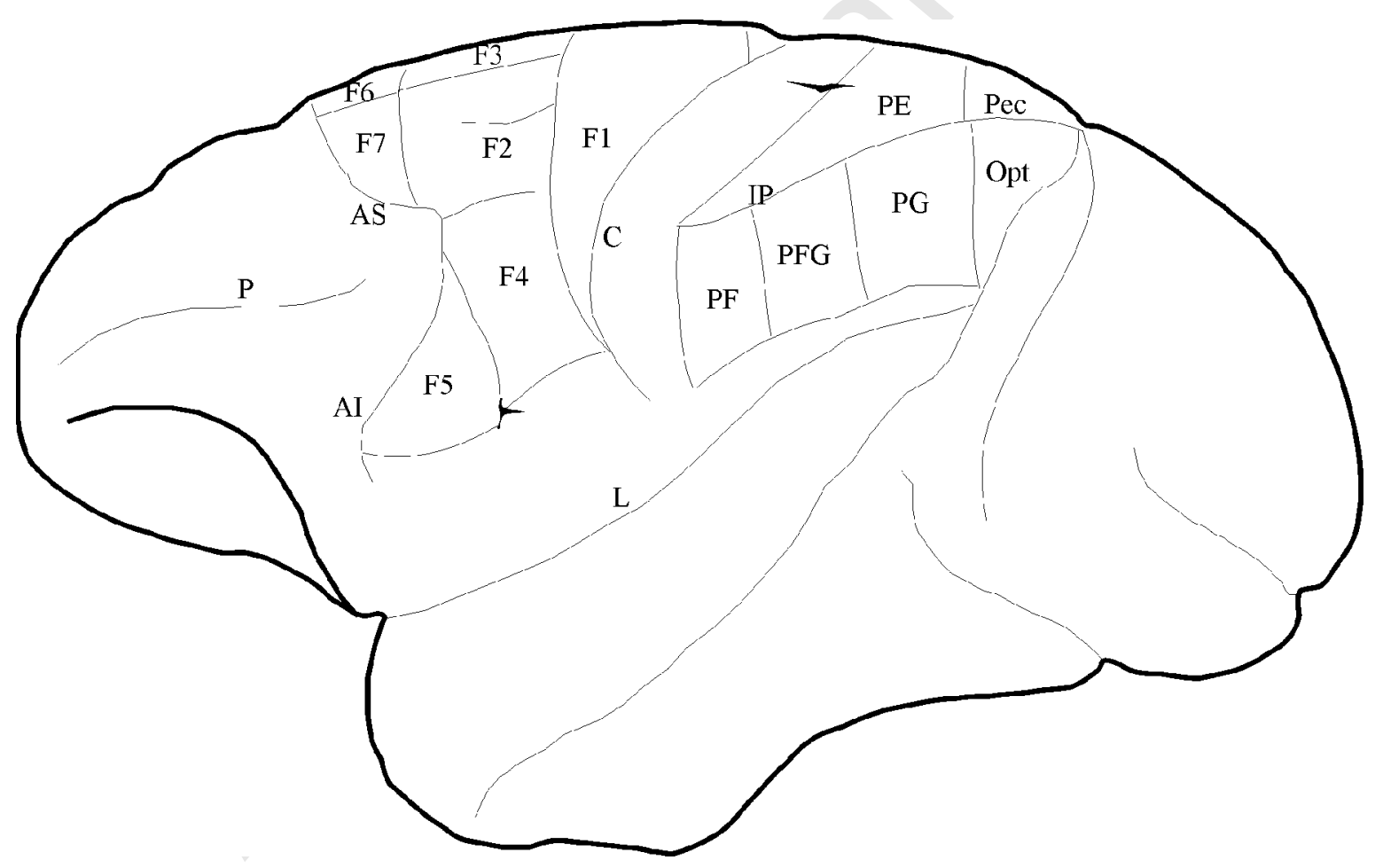

